\title{
Hypothyroidism is associated with higher testosterone levels in postmenopausal women with Hashimoto's thyroiditis
}

\author{
Katica Bajuk Studen, Ajda Biček, Adrijana Oblak, Katja Zaletel, Simona Gaberšček \\ Department of Nuclear Medicine, University Medical Centre, Ljubljana, Slovenia
}

\begin{abstract}
Introduction: There are few data about possible interaction of sex hormones and thyroid autoimmunity and function in women with Hashimoto's thyroiditis (HT) after menopause. Therefore, our aim was to investigate sex hormone levels in euthyroid (EuHT) and hypothyroid (HypoHT) postmenopausal women with HT.

Material and methods: We performed a prospective observational clinical study that included 55 women with HT (AllHT) and 18 healthy subjects (HS) after menopause matched by age, body mass index, follicle-stimulating hormone, and menopause duration. According to their thyrotropin (TSH) level, the AllHT patients were divided into two subgroups: EuHT with TSH in the range 0.35-5.5 mU/L and HypoHT with TSH above $5.5 \mathrm{mU} / \mathrm{L}$. Total and free testosterone (T), sex hormone-binding globulin (SHBG), oestradiol (E2), and progesterone (P) were measured in all subjects. Values are presented as mean \pm SD. The Mann-Whitney U test was used for comparison of values between the groups. Correlations were tested using Kendall's tau test.

Results: In the HypoHT group, significantly higher free T levels were found in comparison to the HS group $(7.89 \pm 3.55$ pmol/L and $7.13 \pm 3.03 \mathrm{pmol} / \mathrm{L}, \mathrm{p}<0.05)$. Furthermore, in HypoHT, free T was significantly higher than in EuHT $(7.19 \pm 5.65 \mathrm{pmol} / \mathrm{L}, \mathrm{p}<0.05)$. SHBG was significantly lower in HypoHT compared with HS (45.4 $\pm 17.4 \mathrm{nmol} / \mathrm{L}$ and $60.09 \pm 19.51 \mathrm{nmol} / \mathrm{L}, \mathrm{p}<0.05)$. No significant correlation was found between sex hormone levels and thyroglobulin and thyroid peroxidase antibodies.

Conclusion: We report significantly higher free and total T levels in hypothyroid postmenopausal women with HT. To our knowledge, this is the first study of sex hormone levels in postmenopausal women with HT. (Endokrynol Pol 2020; 71 (1): 73-75)

Key words: Hashimoto's thyroiditis; testosterone; menopause; hypothyroidism
\end{abstract}

\section{Introduction}

Hashimoto's thyroiditis (HT) is a common autoimmune thyroid disorder, which is more prevalent in women than in men [1]. The female predominance of HT increases sharply during puberty [2], and the prevalence is also higher in patients with polycystic ovary syndrome [3], both suggesting a possible role of sex hormones in the pathogenesis of the disease. After menopause, attenuation of sex hormone influence on autoimmunity would be expected. However, the prevalence of HT in women increases with age [1]. An effect of physiological menopause on circulating androgen levels has not been observed [4]. Several associations between menopause and autoimmune diseases other than HT have been reported [4]. There are no published studies investigating sex hormone levels in euthyroid and hypothyroid women with HT in the menopause. Therefore, our aim was to investigate sex hormone levels in euthyroid and hypothyroid postmenopausal women with HT.

\section{Material and methods}

In this prospective observational clinical study, 55 postmenopausal women with HT and 18 healthy postmenopausal women were included. The protocol of the study was approved by the Republic of Slovenia National Medical Ethics Committee (number 84/05/13). Menopause was confirmed by the absence of menses during the preceding 12-month interval and an elevated follicle-stimulating hormone (FSH) above $20 \mathrm{U} / \mathrm{L}$. Healthy women in the control group had thyrotropin (TSH) in the range $0.35-5.5 \mathrm{mU} / \mathrm{L}$, isoechoic thyroid ultrasound pattern, and normal levels of antibodies against thyroid peroxidase (TPOAb) and thyroglobulin (TgAb). HT was diagnosed by hypoechoic thyroid ultrasound pattern and elevated levels of TPOAb and/or TgAb. According to TSH level, patients with HT (AllHT) were divided into euthyroid HT with TSH in the normal range (EuHT) $(\mathrm{n}=34)$ and hypothyroid HT with TSH level above $5.5 \mathrm{mU} / \mathrm{L}(\mathrm{HypoHT})(\mathrm{n}=21)$. Evaluation of sex hormones included measurement of $\mathrm{FSH}$, total and free testosterone (T), oestradiol (E2), progesterone $(\mathrm{P})$, and sex hormone-binding globulin (SHBG). Women taking hormone replacement therapy for menopausal symptoms or women suffering from any acute infection or other serious illness were not included in the study.

All laboratory measurements were performed in the Laboratory for Clinical Biochemistry and Radiochemistry at the Department of Nuclear Medicine of the University Medical Centre Ljubljana. $\mathrm{TSH}$, free thyroxine $\left(\mathrm{fT}_{4}\right)$, free triiodothyronine $\left(\mathrm{fT}_{3}\right), \mathrm{TPOAb}$, and $\mathrm{TgAb}$ were measured with commercially available kits and the 
ADVIA Centaur System (Siemens Medical Solutions Diagnostics, Dublin, Ireland). FSH, E2, P, total T, and SHBG were measured by chemiluminescent immunometric assay by Immulite $2000 \mathrm{XPi}$ system (Siemens Healthcare Diagnostics, Erlangen, Germany). Free T was calculated from total $\mathrm{T}$ and SHBG.

The data are presented as mean values \pm standard deviation, except for TPOAb and $\mathrm{TgAb}$ where they are presented as median (range). For the comparison of measured values between different groups, the Mann-Whitney U test was used. Correlations were tested using Kendall's tau test. p-values below 0.05 were considered statistically significant.

\section{Results}

The healthy subject (HS) group and the AllHT group were matched by age $(59.8 \pm 3.9$ and $59.6 \pm 4.1$ years, $\mathrm{p}=0.724)$, body mass index (28.5 \pm 4.3 and $26.4 \pm 6.1$, $\mathrm{p}=0.277), \mathrm{FSH}(61.4 \pm 30.2$ and $63.0 \pm 25.2 \mathrm{U} / \mathrm{L}$, $\mathrm{p}=0.668)$, and menopause duration $(10.5 \pm 5.6$ and $9.9 \pm 6.1$ years, $\mathrm{p}=0.623)$. Levels of TPOAb and $\mathrm{TgAb}$ were significantly higher in the AllHT group in comparison to the HS group: 1300 (28-1300) kU/L and 139 (15-500) kU/L) vs. 32 (28-45) kU/L and 15 (15-20) kU/L, respectively, $\mathrm{p}<0.001$ for both. The AllHT group also had significantly higher TSH level than the HS group $(10.7 \pm 18.4$ and $2.78 \pm 1.06 \mathrm{mU} / \mathrm{L}, \mathrm{p}=0.003)$ as well as lower $\mathrm{fT}_{4}$ level $(12.4 \pm 2.6$ and $14.04 \pm 2.34 \mathrm{pmol} / \mathrm{L}$, $\mathrm{p}=0.020)$. The AllHT and HS groups did not differ significantly in $\mathrm{fT}_{3}$ level $(4.51 \pm 0.72$ and $4.82 \pm 0.61$ $\mathrm{pmol} / \mathrm{L}, \mathrm{p}=0.150)$.

Sex hormone levels in the HS and AllHT groups as well as in the EuHT and Hypo HT subgroups are listed in Table I. No significant differences between the HS and AllHT groups were found. However, in the HypoHT subgroup, significantly higher free T levels were found than in the HS group and in the EuHT subgroup. The level of total $\mathrm{T}$ was significantly higher in the HypoHT subgroup than in the EuHT subgroup. SHBG levels were significantly lower in the HypoHT subgroup compared with the HS group. Subgroups did not differ with respect to E2 and P levels (Tab. 1). No significant correlation between sex hormone levels and thyroid antibodies (TgAb and TPOAb) was found.

\section{Discussion}

In this prospective observational clinical study, which included two well-defined groups (HS and AllHT) of postmenopausal women, we found significantly higher free and total T levels in the HypoHT subgroup than in the EuHT subgroup. We also found significantly higher free $\mathrm{T}$ and lower SHBG levels in the HypoHT subgroup than in the HS group. Our observation is not in accordance with the expected anti-inflammatory influence of androgens on the pathogenesis of HT in postmenopausal women [4]. Because we found no significant correlation between higher T levels and thyroid antibodies, we speculate that the higher levels of $\mathrm{T}$ are more likely to be associated with the hypothyroid state than with HT. It was reported that hypothyroidism decreases plasma binding activity of SHBG, which in women results in decreased plasma concentrations of both total $\mathrm{T}$ and $\mathrm{E} 2$, but their unbound fractions are increased [5]. This is in agreement with our finding of higher free T levels in the HypoHT subgroup, but it does not explain the higher total $\mathrm{T}$ in the HypoHT group in comparison to the EuHT group. Of note, studies exploring the association of hypothyroidism with steroid hormone levels were performed several decades ago and were not reproduced with modern laboratory techniques; they also did not report specific data about the period of life of the included subjects (adolescent/adult/postmenopausal/possible PCOS etc.). Significantly lower SHBG levels in the HypoHT subgroup than in the HS group are in agreement with previous reports [5]. We found no association of HT or hypothyroid state with the E2 or P levels in postmenopausal women.

A limitation of our study is a relatively small study sample. Nevertheless, the total and free T levels measured in our study are consistent with age-adjusted population averages and the variance of healthy postmenopausal women. The strengths of our study are its prospective nature, homogeneous well-defined groups of subjects, and analysis of thyroid and sex hormones by modern laboratory techniques.

Table 1. Sex hormone values in healthy subjects and in patients with Hashimoto's thyroiditis. Values are presented as mean \pm standard deviation (SD)

\begin{tabular}{lcccc}
\hline Parameter & HS $(\mathbf{n}=\mathbf{1 8})$ & AllHT $(\mathbf{n}=\mathbf{5 5})$ & EuHT $(\mathbf{n}=\mathbf{3 4})$ & HypoHT $(\mathbf{n}=\mathbf{2 1})$ \\
\hline Total T $[\mathrm{nmol} / \mathrm{L}]$ & $0.77 \pm 0.23$ & $0.78 \pm 0.41$ & $0.78 \pm 0.23$ & $\mathbf{0 . 8 1} \pm \mathbf{0 . 2 8 ^ { \mathrm { b } }}$ \\
\hline Free T [pmol/L] & $7.13 \pm 3.03$ & $7.45 \pm 4.93$ & $7.19 \pm 5.65$ & $\mathbf{7 . 8 9} \pm \mathbf{3 . 5 5 ^ { \mathrm { a } } , \mathrm { b }}$ \\
\hline SHBG $[\mathrm{nmol} / \mathrm{L}]$ & $60.09 \pm 19.51$ & $50.81 \pm 21.49$ & $54.2 \pm 23.3$ & $\mathbf{4 5 . 4} \pm \mathbf{1 7 . 4 ^ { \mathrm { a } }}$ \\
\hline E2 $[\mathrm{nmol} / \mathrm{L}]$ & $0.11 \pm 0.05$ & $0.11 \pm 0.06$ & $0.10 \pm 0.05$ & $0.11 \pm 0.07$ \\
\hline P $[\mathrm{nmol} / \mathrm{L}]$ & $0.64 \pm 0.02$ & $0.69 \pm 0.21$ & $0.65 \pm 0.07$ & $0.75 \pm 0.32$ \\
\hline
\end{tabular}

HS — healthy subjects; AllHT — all patients with Hashimoto's thyroiditis; EuHT — euthyroid patients with Hashimoto's thyroiditis; HypoHT — hypothyroid patients with Hashimoto's thyroiditis; T — testosterone; SHBG — sex hormone-binding globulin; E2 — oestradiol; P — progesterone; ${ }^{\mathrm{p} p}<0.05$ when compared with HS; bp $<0.05$ when compared with EuHT 


\section{Conclusion}

Our finding of significantly higher T levels in hypothyroid than in euthyroid postmenopausal women represents a novelty, which can be partly explained by the lower SHBG levels. Neither T nor other sex hormones seem to be associated with the occurrence of HT.

\section{Funding}

The authors received no specific funding for this work

\section{Conflict of interest}

None.

\section{References}

1. Hollowell JG, Staehling NW, Flanders WD, et al. Serum TSH, T(4), and thyroid antibodies in the United States population (1988 to 1994): National Health and Nutrition Examination Survey (NHANES III). J Clin Endocrinol Metab. 2002; 87(2): 489-499, doi: 10.1210/jcem.87.2.8182, indexed in Pubmed: 11836274.

2. Mariotti S, Prinzis A, Ghiani M, et al. Puberty is associated with a marked increase of the female sex predominance in chronic autoimmune thyroiditis. Horm Res. 2009; 72(1): 52-56, doi: 10.1159/000224341, indexed in Pubmed: 19571560.

3. Janssen OE, Mehlmauer N, Hahn S, et al. High prevalence of autoimmune thyroiditis in patients with polycystic ovary syndrome. Eur J Endocrinol. 2004; 150(3): 363-369, doi: 10.1530/eje.0.1500363, indexed in Pubmed: 15012623

4. Bove R. Autoimmune diseases and reproductive aging. Clin Immunol. 2013; 149(2): 251-264, doi: 10.1016/j.clim.2013.02.010, indexed in Pubmed: 23522436

5. Krassas GE, Poppe K, Glinoer D. Thyroid function and human reproductive health. Endocr Rev. 2010; 31(5): 702-755, doi: 10.1210/er.2009-0041, indexed in Pubmed: 20573783. 